\title{
Rapid Freezing/Freeze Substitution Transmission Electron Microscopy Assessment of Cultured Endothelial Cell Glycocalyx Structure
}

\author{
E.E. Ebong, ${ }^{1,2}$ F.P. Macaluso, ${ }^{3}$ D.C. Spray, ${ }^{2}$ and J.M. Tarbell ${ }^{1}$
}

${ }^{1}$ Department of Biomedical Engineering, CUNY City College of New York, Convent Ave. and $140^{\text {th }}$ Street, New York, NY 10031

2 Department of Neuroscience, Albert Einstein College of Medicine, Bronx, NY, 10461

3 Analytical Imaging Facility, Albert Einstein College of Medicine, Bronx, NY, 10461

\section{OBJECTIVE:}

Recent publications questioned the validity of endothelial cell (EC) culture studies of glycocalyx (GCX) function, due to findings that GCX in vitro may be substantially thinner than in vivo [1]. The assessment of thickness differences is complicated by GCX collapse during dehydration for traditional electron microscopy. We measured in vitro GCX thickness using rapid freezing/freeze substitution transmission electron microscopy (RF/FS-TEM), taking advantage of high spatial resolution provided by TEM and the capability to stably preserve the GCX in its hydrated configuration by RF/FS.

\section{METHODS AND RESULTS:}

Bovine aortic and rat fat pad endothelial cells (BAEC and RFPEC) that have been used for studies of GCX function [2-5] were subjected to conventional- or RF/FS-TEM. Our conventionally preserved BAEC GCX was dense and $\sim 0.040 \mu \mathrm{m}$ in thickness (Fig 1A and 1B). RF/FS-TEM revealed a substantial GCX that had the appearance of a loose fibril mesh and impressive thickness of $\sim 11 \mu \mathrm{m}$ for BAEC (Fig. 1C) and $\sim 5 \mu \mathrm{m}$ for RFPEC (Fig. 1D).

We also assessed the utility of RF/FS-TEM for detecting differences in the GCX depending on environmental changes that affect GCX composition, which includes proteoglycans, glycosaminoglycans, glycoproteins, and plasma proteins. GCX thickness was unaffected but ultrastructural modification occurred due to treatment of RF/FS processed BAEC with heparinase III enzyme at a $15 \mathrm{mU} / \mathrm{mL}$ concentration (that degrades $50 \%$ of the GCX heparan sulfate proteoglycan and significantly blocks GCX function [2-5]). Removal of protein from EC culture media, to collapse the GCX, severely reduced BAEC and RFPEC GCX thickness to levels that were not visible by RF/FS-TEM (Fig. 1E). These results demonstrate the effectiveness of using RF/FS-TEM to reveal GCX modifications in response to micro-environmental changes such as protein content modification and enzymatic activity.

We experienced that RF/FS TEM was limited by the fact that post-RF/FS immunogold labeling techniques adversely affected cell morphology and pre-RF/FS use of traditional immunogold or novel immuno-nanogold/silver enhancement labeling caused ice damage and subsequent loss of the GCX and other cellular structures. Therefore, immunoconfocal microscopy was used to supplement $\mathrm{RF} / \mathrm{FS}$-TEM. Immunoconfocal studies confirmed that the in vitro GCX is comprised of extensive and well integrated heparan sulfate proteoglycan, hyaluronic acid proteoglycan, and protein layers. The GCX was found to be $\sim 1.4$ to $\sim 4.3 \mu \mathrm{m}$ thick as previously reported in vitro using confocal microscopy [6]. The revelation of a lesser GCX thickness by immunoconfocal microscopy is most likely due to the fact that paraformaldehyde/glutaraldehyde preservation for confocal microscopy cross-links the GCX components and subsequently pulls the GCX inward towards the cell surface. 


\section{CONCLUSIONS:}

New observations by RF/FS-TEM, taken together with immunoconfocal studies, provide a new life-like perspective of the GCX on cultured EC as a fibril mesh with well integrated components and substantial thickness. This work supports the continued use of cultured EC for fundamental studies of GCX and its function in the vasculature.

\section{References}

[1] Potter DR and Damiano ER, Circ Res 2008;102(7):770-776.

[2] Lopez-Quintero SV et al., Am J Physiol Heart Circ Physiol 2009;296(5):H1451-1456.

[3] Pahakis MY et al., Biochem Biophys Res Commun 2007;355(1):228-233.

[4] Thi MM et al., Proc Natl Acad Sci U S A 2004;101(47):16483-16488.

[5] Yao Y et al., Am J Physiol Heart Circ Physiol 2007;293(2):H1023-1030.

[6] Barker AL et al., Physical chemistry chemical physics 2004;6:1006-1011.

\section{$\dagger$ Acknowledgment}

This work was supported by the National Institutes of Health Grants 5T32HL007675, HL73732, HL57093, and HL094889. We acknowledge the Albert Einstein College of Medicine (AECOM) Analytical Imaging Facility (AIF) for assistance with the TEM studies, and thank the AECOM Cellular and Molecular Neuroimaging Core for confocal microscope use.

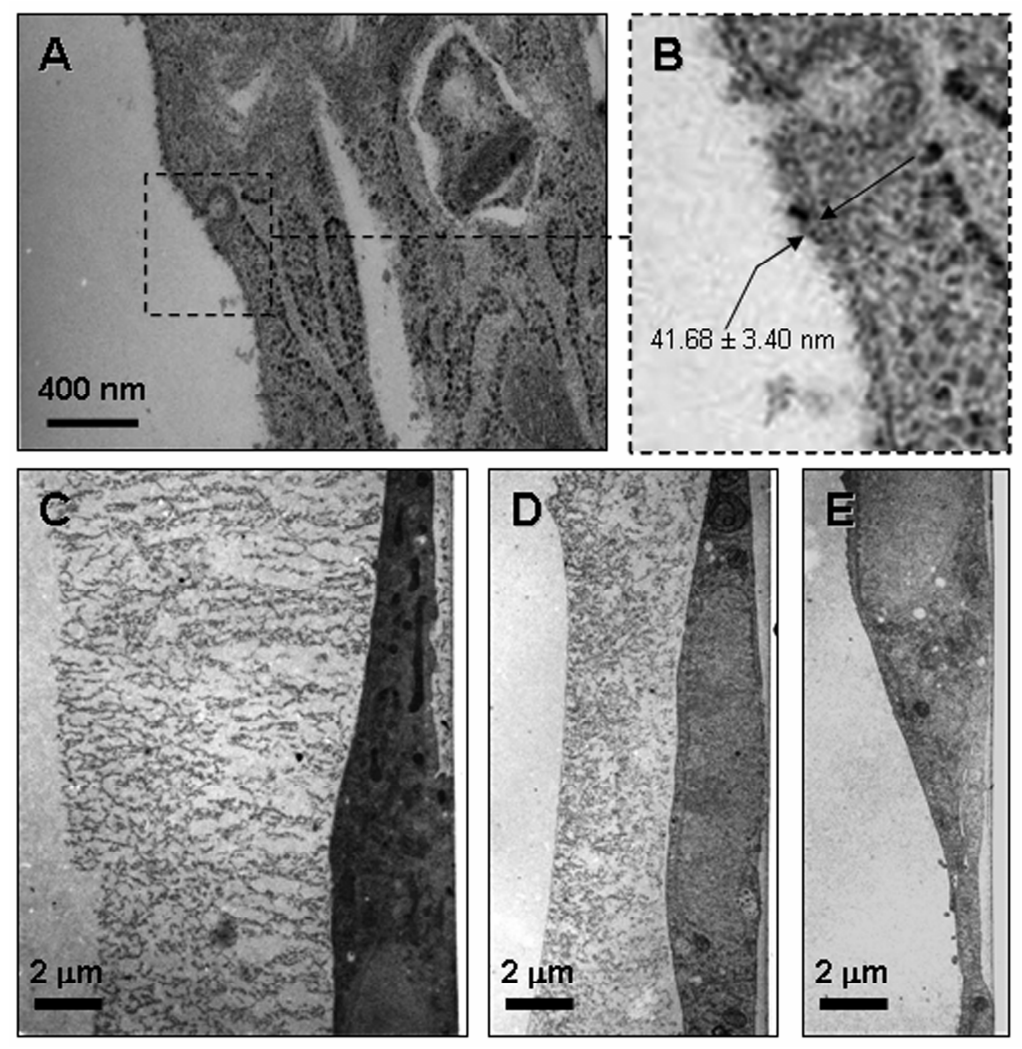

FIG. 1. TEM of GCX-covered EC. (A) BAEC: conventionally preserved, ruthenium red and osmium tetroxide labeled, and alcohol dehydrated. (B) Expanded region of conventionally processed BAEC. (C) BAEC and (D) RFPEC: RF/FS preserved and osmium tetroxide stained. (E) GCX was not expressed on BAEC when FBS and BSA were removed. 